\title{
Influence of Rotation Increments on Imaging Performance for a Rotatory Dual-Head PET System
}

\author{
Fanzhen Meng, Xu Cao, Xuezhou Cao, Jianxun Wang, Liang Li, \\ Xueli Chen, Shouping Zhu, and Jimin Liang \\ Engineering Research Center of Molecular and Neuro Imaging of Ministry of Education, School of Life Science and Technology, \\ Xidian University, Xian, Shaanxi 710071, China \\ Correspondence should be addressed to Shouping Zhu; spzhu@xidian.edu.cn and Jimin Liang; jiminliang@gmail.com
}

Received 22 August 2016; Revised 15 November 2016; Accepted 7 December 2016; Published 5 January 2017

Academic Editor: Enzo Terreno

Copyright (c) 2017 Fanzhen Meng et al. This is an open access article distributed under the Creative Commons Attribution License, which permits unrestricted use, distribution, and reproduction in any medium, provided the original work is properly cited.

For a rotatory dual-head positron emission tomography (PET) system, how to determine the rotation increments is an open problem. In this study, we simulated the characteristics of a rotatory dual-head PET system. The influences of different rotation increments were compared and analyzed. Based on this simulation, the imaging performance of a prototype system was verified. A reconstruction flowchart was proposed based on a precalculated system response matrix (SRM). The SRM made the relationships between the voxels and lines of response (LORs) fixed; therefore, we added the interpolation method into the flowchart. Five metrics, including spatial resolution, normalized mean squared error (NMSE), peak signal-to-noise ratio (PSNR), contrast-to-noise (CNR), and structure similarity (SSIM), were applied to assess the reconstructed image quality. The results indicated that the $60^{\circ}$ rotation increments with the bilinear interpolation had advantages in resolution, PSNR, NMSE, and SSIM. In terms of CNR, the $90^{\circ}$ rotation increments were better than other increments. In addition, the reconstructed images of $90^{\circ}$ rotation increments were also flatter than that of $60^{\circ}$ increments. Therefore, both the $60^{\circ}$ and $90^{\circ}$ rotation increments could be used in the real experiments, and which one to choose may depend on the application requirement.

\section{Introduction}

Dual-head positron emission tomography (PET) systems have emerged in recent years at a lower cost and complexity [1-5]. For a stationary dual-head PET system $[3,6-8]$, the incomplete angle information degrades the spatial resolution perpendicular to the detector heads even if the iteration algorithms are used [9-12]. Some researchers have adopted the rotation operation to solve this problem [13-15]. There are mainly two schemes, rotating the detectors or rotating the object. For example, in 2006, Bruyndonckx et al. have proposed a prototype PET scanner in which the two detector heads are installed on a rotating platform [16]. In 2010, a small-animal PET system including a rotation platform in the middle of the detector heads has been addressed [2]. Nevertheless, how to determine the rotation increments is also an open problem. Efthimiou et al. have evaluated the performance of a rotatory dual-head system by a series of angles [17], and in their work the system response matrix (SRM) is geometrically estimated which is not accurate and is apt to cause depth of interaction (DOI) blurring near the field of view (FOV) boundaries $[8,13,17]$.

The quality of the reconstructed images depends on the accurate modeling of relationships between the object space and measurement space [18]. The Monte Carlo (MC) simulation can accurately simulate the system model through a feasible empirical setup [19], but the SRM is precalculated and prestored in the computer. For the precalculated SRM, the relationships between the voxels (object space) and lines of response (LORs) (measurement space) are fixed. Once the system rotates an angle, the FOV should be rotated by the same angle to match with the precalculated SRM. When the rotation angle is not a multiple of $90^{\circ}$, an image interpolation operation is needed to build the relationships of the reconstructed images before and after rotation. In this 


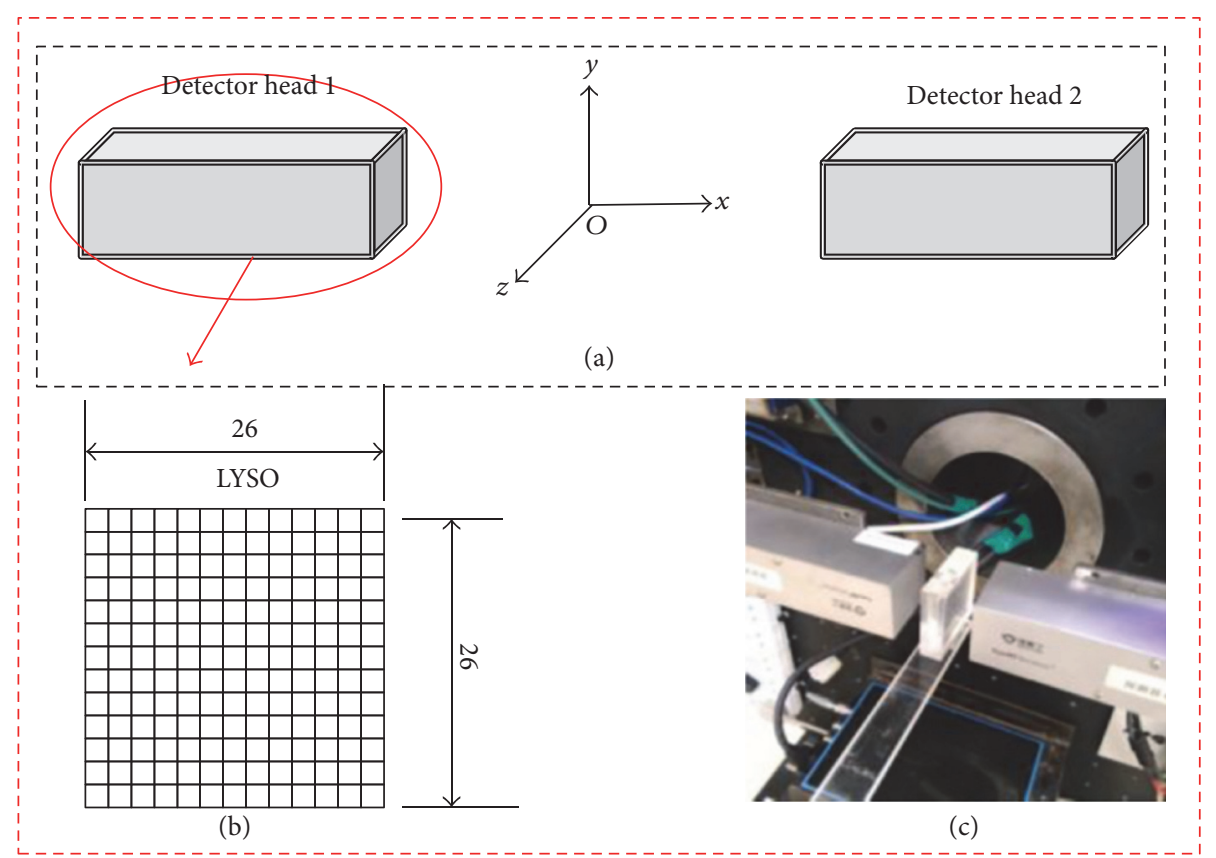

Figure 1: System structure of the dual-head PET system. (a) Geometry structure of the system and coordinate system. (b) Crystals of the system. (c) Prototype system.

study, we incorporate the interpolation methods [20] including the nearest neighbor interpolation, bicubic interpolation, and bilinear interpolation into the iteration reconstruction process. The different interpolation methods are compared to find the optimal interpolation, whose errors have minimum influence on the optimization cost function.

The purpose of this study is to analyze the influences of different rotation increments on the imaging performances of a rotatory dual-head PET system based on an accurate SRM. The rest of the article is organized as follows. In Section 2, we introduce the simulation of the dual-head PET system and describe the reconstruction method and experiment schemes. The results are shown in Section 3. Finally, the discussion and conclusion are in Section 4.

\section{Materials and Methods}

\subsection{Simulation Scheme}

2.1.1. Simulation of Dual-Head PET System. We used the software package of GATE $6.2[21,22]$ to simulate the characteristics of a dual-head PET system. Two pixelated planar detector heads were designed in opposing positions. Each detector head contained $26 \times 26$ LYSO crystals with the size of $1.89 \times 1.89 \times 13 \mathrm{~mm}^{3}$. The pitch of each crystal was $2.038 \mathrm{~mm}$. The system geometry and coordinate system are shown in Figures 1(a)-1(b). The $x$-axis is vertical to the detector heads and the $y$-axis is vertical to the ground. The other direction is defined as the $z$-axis, which is also the rotation axis. In this study, the system parameters were based on the prototype system built in our lab, as shown in Figure 1(c).
TABLE 1: Rotation increments and corresponding scanning positions.

\begin{tabular}{lc}
\hline Rotation increments & Scanning positions \\
\hline $0^{\circ}$ & $0^{\circ}$ \\
$90^{\circ}$ & $0^{\circ} \rightarrow 90^{\circ}$ \\
$60^{\circ}$ & $0^{\circ} \rightarrow 60^{\circ} \rightarrow 120^{\circ}$ \\
$45^{\circ}$ & $0^{\circ} \rightarrow 45^{\circ} \rightarrow 90^{\circ} \rightarrow 135^{\circ}$ \\
$36^{\circ}$ & $0^{\circ} \rightarrow 36^{\circ} \rightarrow 72^{\circ} \rightarrow 108^{\circ} \rightarrow 144^{\circ}$ \\
\hline
\end{tabular}

The back-to-back sources, where the two annihilation photons were generated at $180^{\circ}$, were used in the simulation. All sources were monoenergetic and no radioactive decays were considered. The energy resolution was set to $15 \%$ at $511 \mathrm{keV}$. The energy window was set from 350 to $650 \mathrm{keV}$ and the timing coincidence window was $10 \mathrm{~ns}$.

2.1.2. Rotation Description. In the simulation, the rotation was carried out in a step-and-shoot mode and four different rotation increments were considered. The rotation increments $\left(90^{\circ}, 60^{\circ}, 45^{\circ}\right.$, and $\left.36^{\circ}\right)$ and the corresponding scanning positions were listed in Table 1 . The separation distance between the two heads also could affect the imaging performance of the system. In order to compare the effects of the separation distance, we set the distance at $50 \mathrm{~mm}, 70 \mathrm{~mm}$, and $100 \mathrm{~mm}$, respectively. Furthermore, we calculated the distances which would keep the detector heads from overlapping in space. The calculated results were shown in Figure 2.

2.1.3. Phantom Description. Three types of phantoms (point phantom, hot spot phantom, and Derenzo phantom) were 




(a)

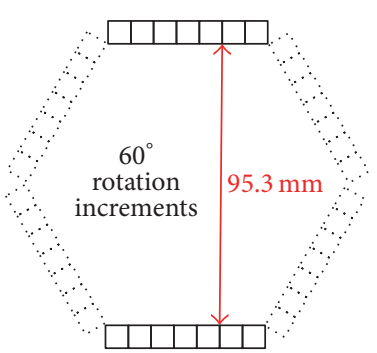

(b)

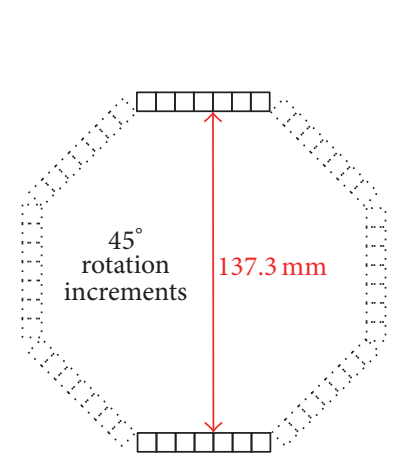

(c)

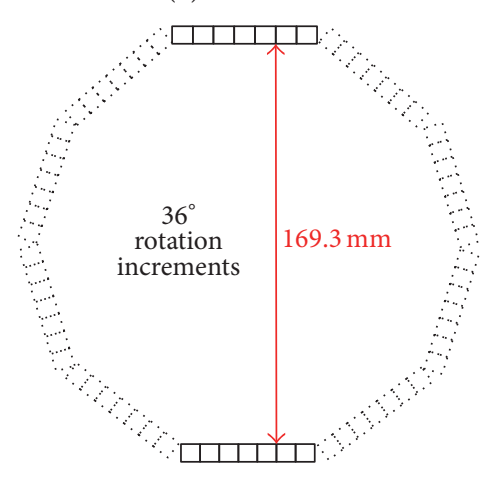

(d)

FIGURE 2: Distances between two nonoverlapping detector heads in space.

TABle 2: Total coincidence events of different distances for phantoms.

\begin{tabular}{lccc}
\hline & $50 \mathrm{~mm}$ & $70 \mathrm{~mm}$ & $100 \mathrm{~mm}$ \\
\hline Point phantom & $3.4 \times 10^{5}$ & $2.2 \times 10^{5}$ & $1.3 \times 10^{5}$ \\
Hot spot phantom & $2.4 \times 10^{7}$ & $1.4 \times 10^{7}$ & $8.9 \times 10^{6}$ \\
Derenzo phantom & $1.0 \times 10^{7}$ & $6.8 \times 10^{6}$ & $4.2 \times 10^{6}$ \\
\hline
\end{tabular}

utilized to assess the effect of different rotation increments, and their structures were shown in Figure 3. The total coincidence events of different distances for these phantoms were listed in Table 2.

Point Phantom. Three-point sources with a diameter of $0.5 \mathrm{~mm}$, which were denoted as P1, P2, and P3 in Figure 3(a), were simulated to characterize the spatial resolution. These sources were placed at $0 \mathrm{~mm}, 5 \mathrm{~mm}$, and $10 \mathrm{~mm}$ away from the center of the (FOV) in the $y$-direction, respectively.

Hot Spot Phantom. As shown in Figure 3(b), six hot spots with a diameter of $0.5 \mathrm{~mm}, 1.0 \mathrm{~mm}, 1.5 \mathrm{~mm}, 2 \mathrm{~mm}, 2.5 \mathrm{~mm}$, and $3.0 \mathrm{~mm}$ were inserted into the cold background phantom. The phantom was $0.5 \mathrm{~mm}$ in height and all the spots were $7 \mathrm{~mm}$ away from the FOV center.

Derenzo Phantom. The Derenzo phantom included in GATE was used in our simulation. It contained 5 groups of hot rods with a diameter of $1.0 \mathrm{~mm}, 1.2 \mathrm{~mm}, 1.5 \mathrm{~mm}, 2.0 \mathrm{~mm}$, and $2.5 \mathrm{~mm}$, respectively, as shown in Figure 3(c). The overall diameter of the phantom was $30 \mathrm{~mm}$ and $0.5 \mathrm{~mm}$ in height.
During the simulation, the phantom was placed in the center of the FOV and its axis was parallel to the detector heads.

2.2. Reconstruction for the Rotatory Dual-Head PET System. In order to describe the transmission of the $\gamma$ rays accurately, we calculated the system response matrix by MC simulations with GATE. More specifically, the voxel-based symmetry properties of the dual-head PET configuration were explored to reduce the simulation time [23]. The readers could consult our previous work [7] for details.

The precalculated SRM fixed the relationships between the voxels and the LORs. When the system was rotated by an angle, the reconstructed images were in need of rotating by the same angle to match the precalculated SRM. In order to fuse the multiangle data together, we proposed a reconstruction scheme as shown in Figure 4. We supposed that the rotation increment was $\theta$ and the scanning position number was $N$, where $N \theta=180$. Thus, we divided the scanning data into $N$ groups based on the scanning position. For each group, the ordered subset expectation maximization (OSEM) [24] with five subsets was applied to reconstruction. After the reconstruction of one group was finished, we rotated the slices perpendicular to the rotation axis $\theta^{\circ}$ and then the rotated results acted as the initial matrix of reconstruction for the next group data. At the end of each iteration, the rotated angles were adjusted to $-\left(180^{\circ}-\theta^{\circ}\right)$ to match with the reconstructed images of the first group data. Then, the iterative reconstruction would be stopped if the end condition was met; otherwise the $N$ group data were reconstructed again.

When an image was rotated some angles which were not a multiple of $90^{\circ}$, an interpolation method was needed. 


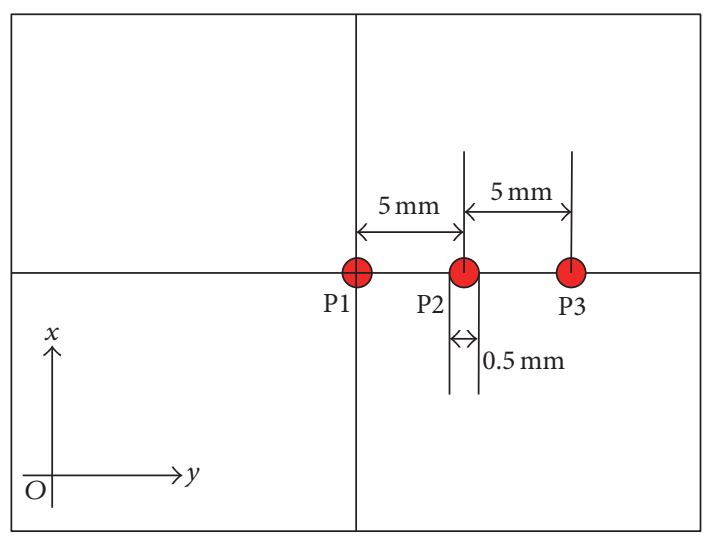

(a)

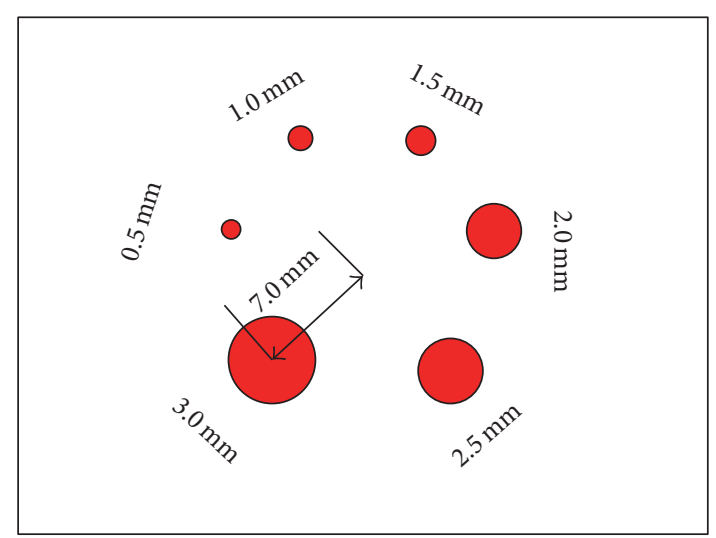

(b)

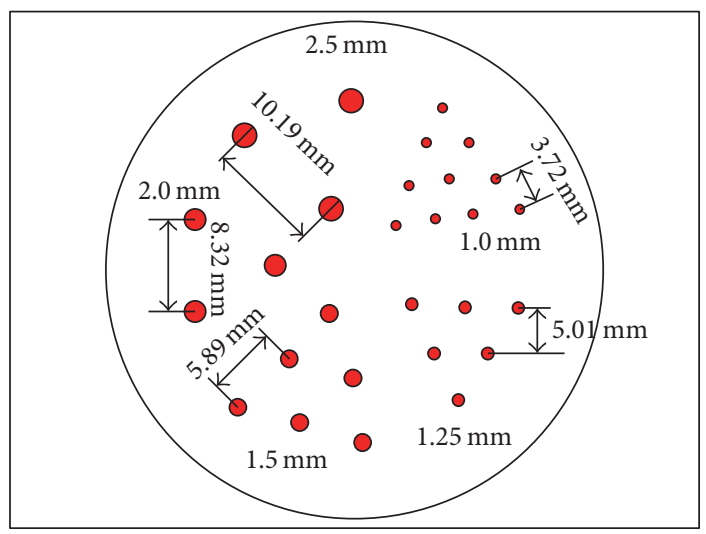

(c)

Figure 3: Simulation phantoms. (a) Three-point sources phantom. (b) Hot spot phantom. (c) Derenzo phantom.

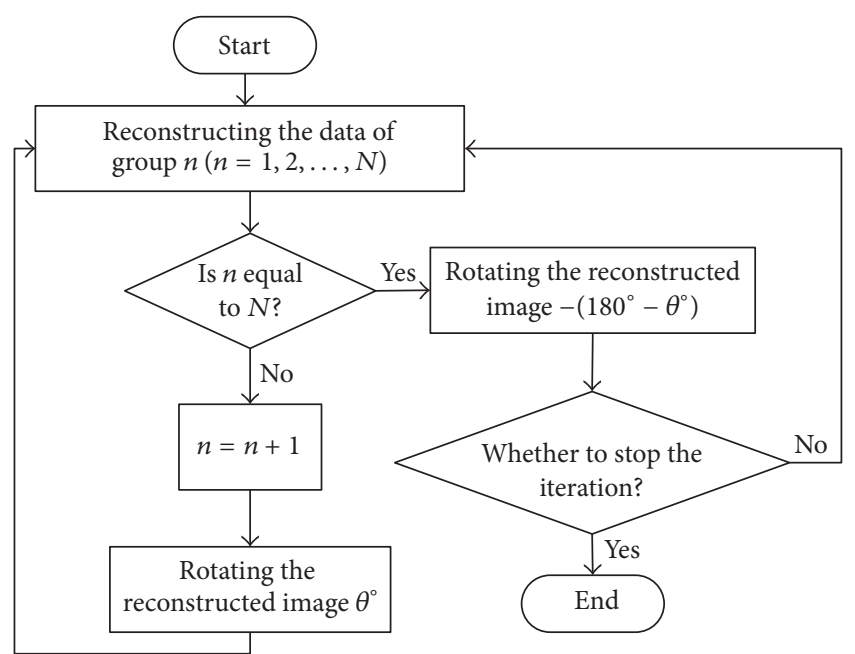

FIGURE 4: Main reconstruction flowchart.

In this study, we applied the nearest interpolation, bilinear interpolation, and bicubic interpolation.

2.3. Image Quality Assessment. Five metrics, including spatial resolution, normalized mean squared error (NMSE), peak signal-to-noise ratio (PSNR), contrast-to-noise ratio (CNR), and structure similarity (SSIM), were applied to assess the quality of the reconstructed images.

The SSIM index measured the similarity between two images $[25,26]$. The spatial resolution was characterized by the full width at half maximum (FWHM) [27] of the point sources. The NMSE [28] was defined as follows:

$$
\mathrm{NMSE}=\frac{\sum_{m=0}^{M-1} \sum_{n=0}^{N-1}(f(m, n)-t(m, n))^{2}}{\sum_{m=0}^{M-1} \sum_{n=0}^{N-1} t(m, n)},
$$

where $f(m, n)$ and $t(m, n)$ were the pixel values of the reconstructed image and true image, respectively. $M$ and $N$ were the number of rows and columns in the images.

The PSNR [29] was calculated as follows:

\section{PSNR}

$$
=10 \log _{10} \frac{\operatorname{MAX}^{2}}{(1 / M N) \sum_{m=0}^{M-1} \sum_{n=0}^{N-1}\|f(m, n)-t(m, n)\|^{2}} .
$$

Herein, MAX was the maximum possible pixel value.

In $(3)$, the CNR $[30,31]$ was defined. $\mu_{\text {roi }}$ and $\mu_{\text {back }}$ denoted the mean value of the ROI and background in the 


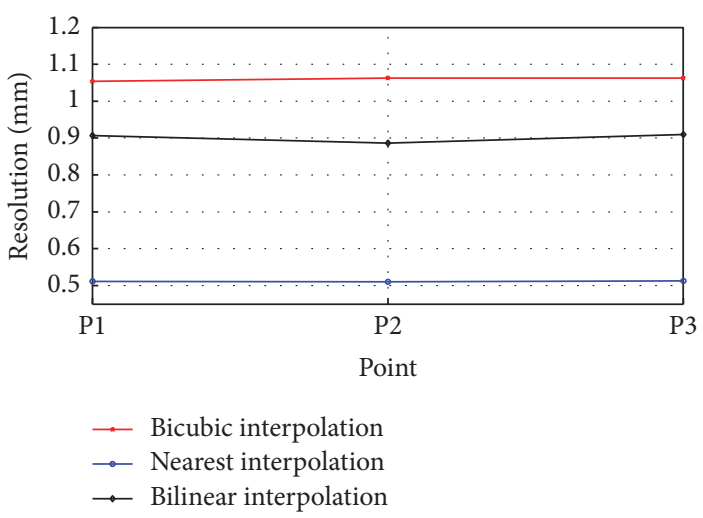

(a)

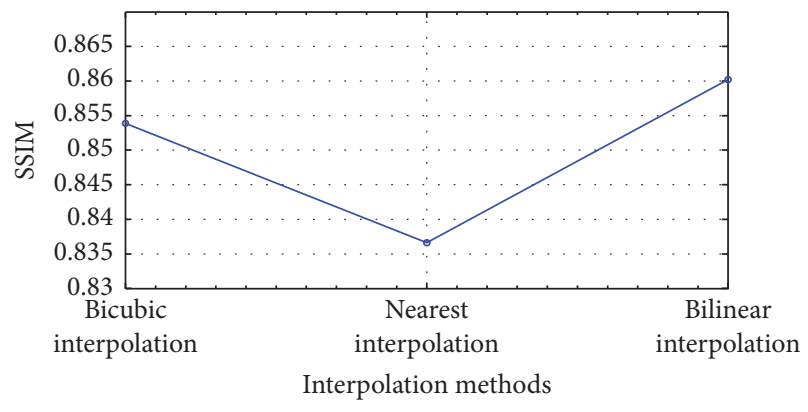

(b)

FIGURE 5: Resolution and SSIM with 45 rotation increments for different interpolation methods. (a) The results of resolution. (b) The results of SSIM.

reconstructed image, respectively; $\delta_{\text {roi }}$ and $\delta_{\text {back }}$ denoted their standard deviations.

$$
\mathrm{CNR}=\frac{\left|\mu_{\text {roi }}-\mu_{\text {back }}\right|}{\sqrt{\left(\delta_{\text {roi }}^{2}+\delta_{\text {back }}^{2}\right) / 2}} .
$$

\section{Results}

For all experiments, the size of the reconstructed image matrix was $64 \times 64 \times 64$ with a cubic voxel of $0.5 \mathrm{~mm} \times$ $0.5 \mathrm{~mm} \times 0.5 \mathrm{~mm}$. Therefore, the entire reconstructed FOV was $32 \times 32 \times 32 \mathrm{~mm}^{3}$.

3.1. Accuracy of Different Interpolation Methods. In this subsection, we compared the performance of the different interpolation methods based on the point phantom and hot phantom. The reconstructed point phantom image provided the spatial resolution information, and the hot phantom was used to calculate the SSIM. For simplicity, we only used data simulated in the condition of $45^{\circ}$ rotation increments with $100 \mathrm{~mm}$ distance between the two detectors.

As shown in Figure 5(a), the nearest interpolation method achieves the higher spatial resolution than the other two interpolation methods. In Figure 5(b), the bilinear interpolation method yields the highest SSIMs. Compared with the other interpolation methods, the bicubic interpolation performed poorly in both resolution and SSIM. Therefore, we only used and compared the nearest and bilinear interpolation in Sections 3.2 and 3.3.

3.2. Resolving Capability. The system's resolving capability was verified based on two phantoms: point phantom and Derenzo phantom. The point phantom was utilized to afford the quantitative evaluations, and the Derenzo phantom was mainly used to provide the qualitative assessments. In the quantitative analysis, five rotation increments were considered with three distances $(50 \mathrm{~mm}, 70 \mathrm{~mm}$, and $100 \mathrm{~mm})$, and only the nearest interpolation method was used. In the qualitative analysis, the reconstructed results of $90^{\circ}$ and $60^{\circ}$ rotation increments were displayed. Furthermore, the results
TABLE 3: Resolutions with different rotation increments with the nearest interpolation.

\begin{tabular}{llllll}
\hline & $0^{\circ}$ & $90^{\circ}$ & $60^{\circ}$ & $45^{\circ}$ & $36^{\circ}$ \\
\hline \multicolumn{7}{c}{$100 \mathrm{~mm}$} \\
P1 & 3.93 & 0.68 & 0.51 & 0.65 & 0.61 \\
P2 & 3.95 & 0.71 & 0.62 & 0.66 & 0.62 \\
P3 & 4.01 & 0.69 & 0.51 & 0.66 & 0.63 \\
\hline \multicolumn{7}{c}{$70 \mathrm{~mm}$} \\
P1 & 3.16 & 0.71 & 0.48 & 0.59 & 0.55 \\
P2 & 3.15 & 0.71 & 0.49 & 0.61 & 0.56 \\
P3 & 3.18 & 0.72 & 0.48 & 0.60 & 0.56 \\
\hline \multicolumn{7}{c}{$50 \mathrm{~mm}$} \\
P1 & 3.04 & 0.79 & 0.52 & 0.69 & 0.65 \\
P2 & 3.04 & 0.78 & 0.53 & 0.69 & 0.65 \\
P3 & 3.08 & 0.80 & 0.54 & 0.71 & 0.66 \\
\hline
\end{tabular}

using the nearest and bilinear interpolation were compared in the condition of $60^{\circ}$ rotation increments.

3.2.1. Resolution Based on the Point Phantom. As the rotation operation mainly made the spatial resolution perpendicular to the detector heads ( $x$-direction) improve greatly, we only calculated and compared the spatial resolutions along $x$-direction. The spatial resolutions of points $\mathrm{P} 1, \mathrm{P} 2$, and $\mathrm{P} 3$ are listed in Table 3 . The nonrotated results $\left(0^{\circ}\right)$ were regarded as the reference to illustrate the advantages of the rotation operation. The results show that using the $60^{\circ}$ rotation increments achieves the best spatial resolution. The resolutions increase about $0.15 \mathrm{~mm} \sim 0.25 \mathrm{~mm}$ compared with that of the $90^{\circ}$ and $45^{\circ}$ rotation increments. In addition, the resolution of $36^{\circ}$ and $45^{\circ}$ rotation increments are almost similar to each other. Therefore, the $36^{\circ}$ rotation increments would not be considered in the following sections to simplify the experiments.

3.2.2. Reconstruction Results of the Derenzo Phantom. Figure 6 displays the reconstructed images of the Derenzo 

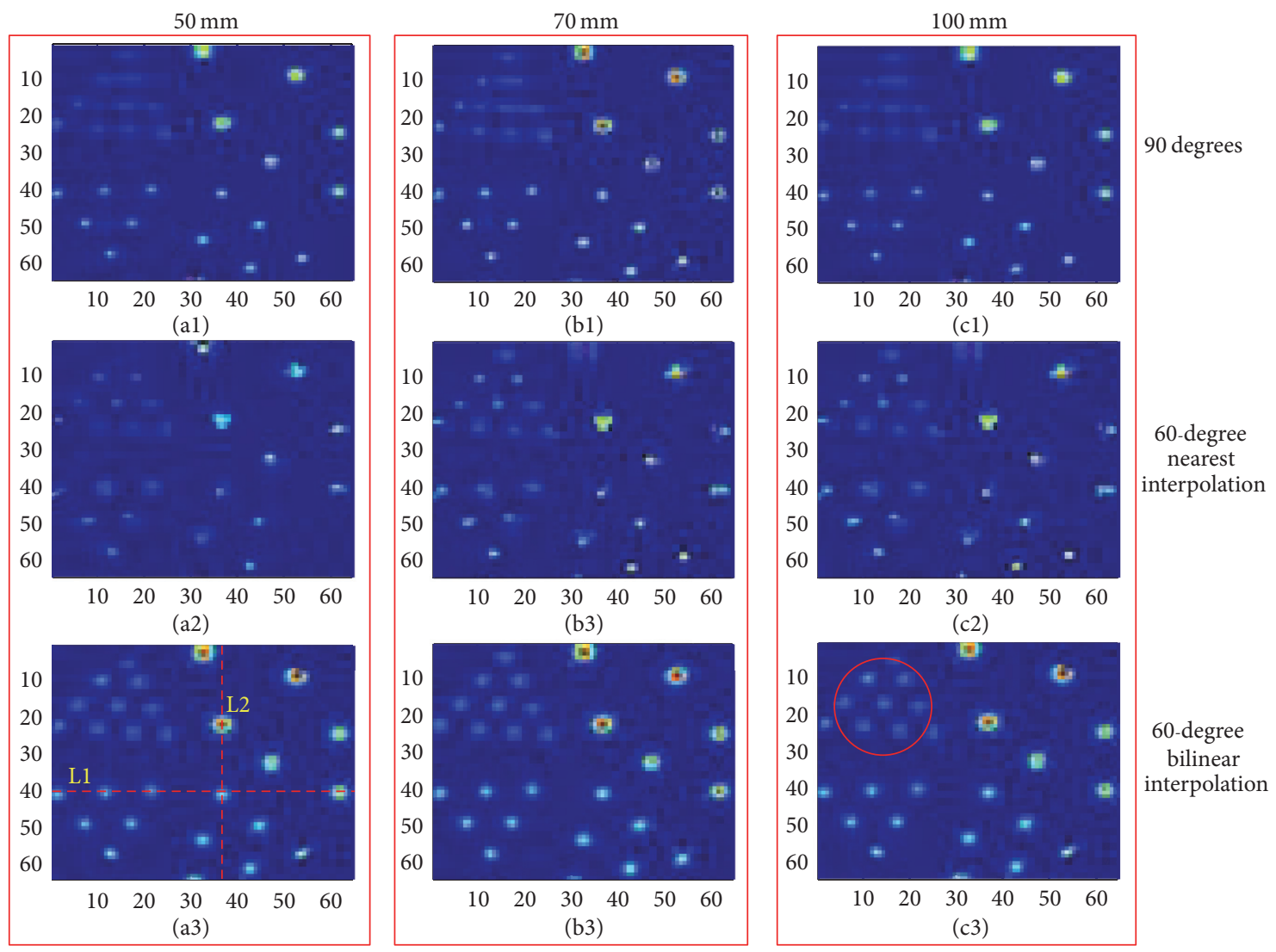

FIGURE 6: Reconstructed images of the Derenzo phantom. Images in the first, second, and third rows are reconstructed with $90^{\circ}$ rotation increments and $60^{\circ}$ rotation increments based on the nearest interpolation and bilinear interpolation, respectively. The columns, from the left to right, show the images from $50 \mathrm{~mm}, 70 \mathrm{~mm}$, and $100 \mathrm{~mm}$.

phantom. Images in the first, second, and third rows are reconstructed with $90^{\circ}$ rotation increments and $60^{\circ}$ rotation increments based on the nearest interpolation and bilinear interpolation, respectively. The columns, from the left to right, show the images from $50 \mathrm{~mm}, 70 \mathrm{~mm}$, and $100 \mathrm{~mm}$. We can find that the results of $60^{\circ}$ rotation increments performed better than the results of $90^{\circ}$ in terms of the resolving capability, especially in the region marked by the red circle. However, the results of $60^{\circ}$ rotation increments with the nearest interpolation show an obvious image distortion, which is not shown in the bilinear interpolation method. Furthermore, we normalized the image intensity along the two red lines in Figure 6(cl) and drew the corresponding profiles in Figure 7. The profiles further illustrated that the nearest interpolation was not a suitable choice for the rotatory PET system.

3.3. Noise Level. In Section 3.2, the system's resolving capability was compared between different rotation increments. In this subsection, we further discussed the noise level for different rotation increments in terms of NMSE, PSNR, CNR, and SSIM. The hot spot phantom with three rotation increments $\left(90^{\circ}, 60^{\circ}\right.$, and $\left.45^{\circ}\right)$ was considered for three distances $(50 \mathrm{~mm}, 70 \mathrm{~mm}$, and $100 \mathrm{~mm})$. The noise levels of the reconstructed images using the nearest and bilinear interpolation were evaluated.

The results are shown in Figure 8. From the view of the interpolation method, the nearest interpolation method has no advantage over the bilinear interpolation in all of the metrics. In view of the rotation increments, the results of the $45^{\circ}$ rotation increments are slightly worse than those of the $60^{\circ}$ rotation increments except for the $\mathrm{CNR}$, but the difference is not obvious. The results of the $90^{\circ}$ rotation increments are more advantageous in CNR than for the $60^{\circ}$ rotation increments, but they have no advantages in terms of PSNR, NMSE, and SSIM.

Furthermore, the reconstructed images of the hot spot phantom are displayed in Figure 9. The first row is the reconstructed results of $90^{\circ}$ rotation increments. The second and the third row are the results of $60^{\circ}$ rotation increments based on the nearest interpolation and bilinear interpolation, respectively. The columns, from the left to right, show the images from $50 \mathrm{~mm}, 70 \mathrm{~mm}$, and $100 \mathrm{~mm}$. The profile curves along the red line in Figure 9(a1) are shown in Figure 10. From the profile curves, it can be observed that the profile curves of $90^{\circ}$ rotation increments are uniform compared to that of $60^{\circ}$ rotation increments.

Overall, the $60^{\circ}$ and $90^{\circ}$ rotation increments had advantages over the other increments. The reconstructed images 

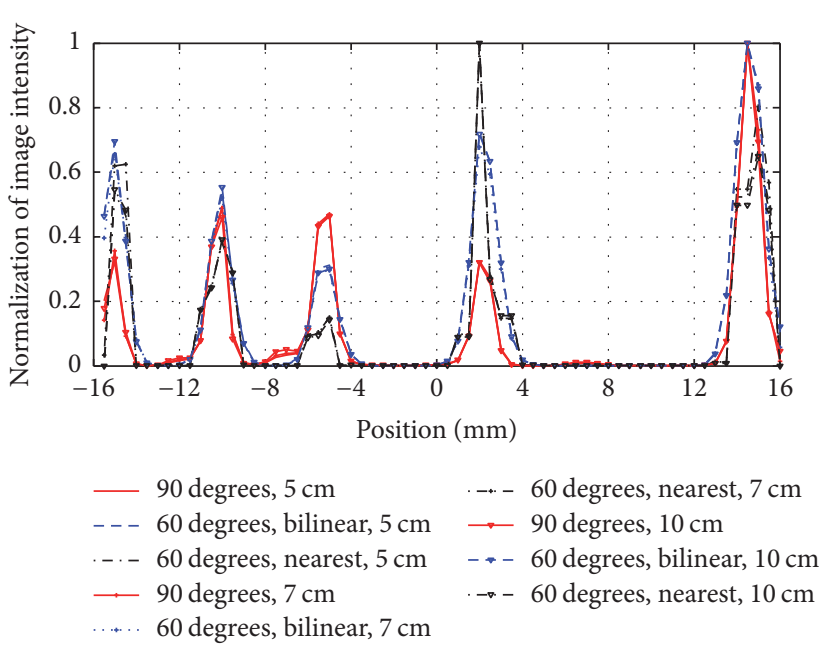

(a)

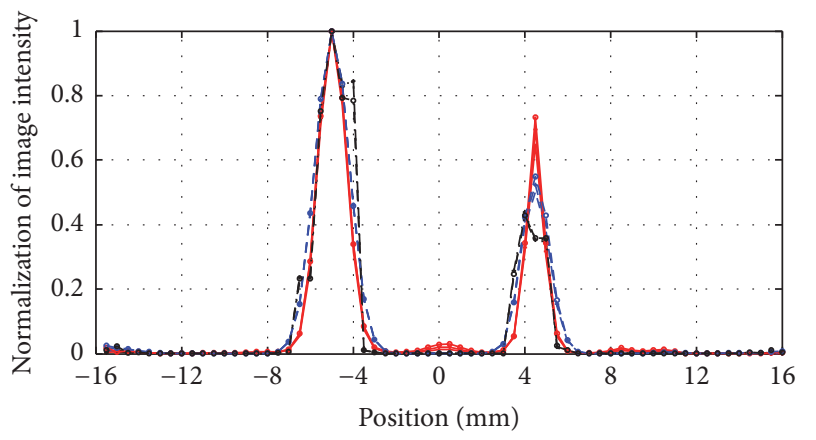

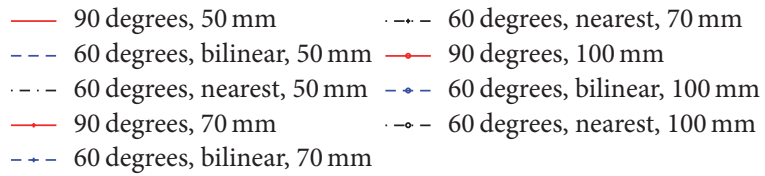

(b)

Figure 7: Profile curves along the red line in Figure 6(a1). (a) The profile along line L1. (b) The profile along line L2.

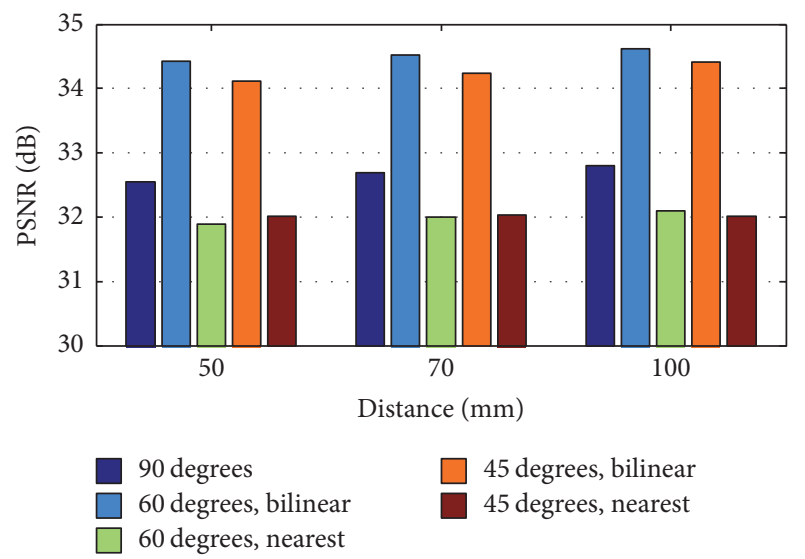

(a)

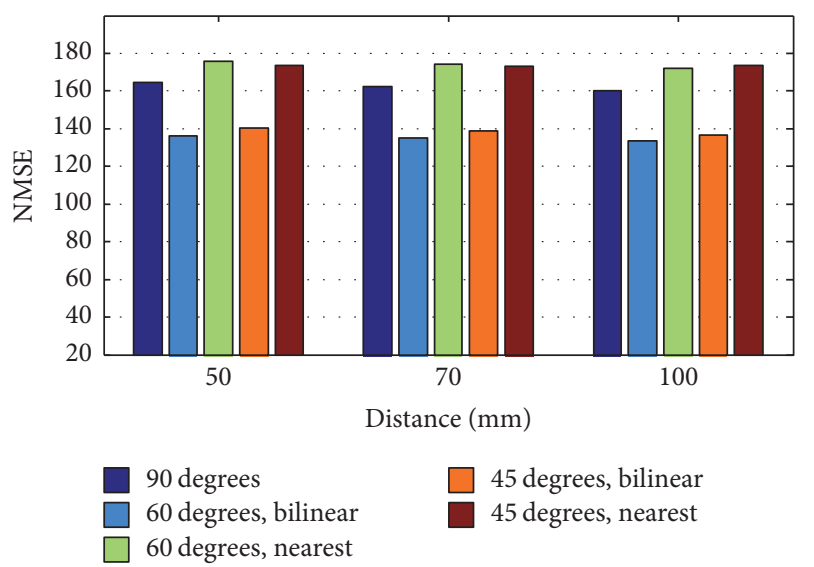

(c)

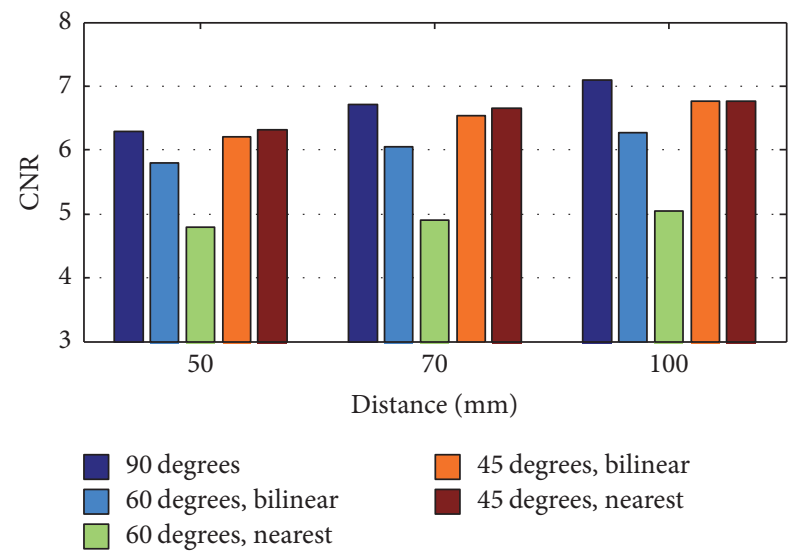

(b)

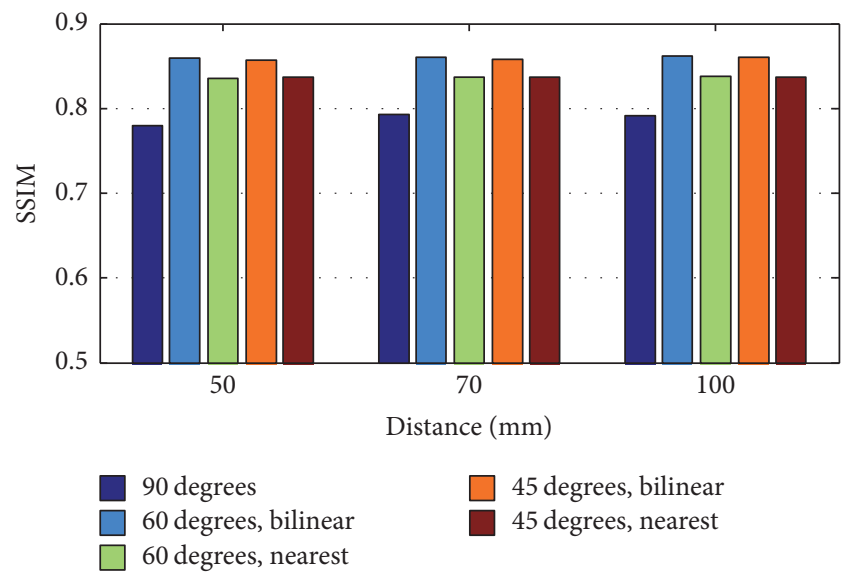

(d)

FIGURE 8: Results of different rotation increments with bilinear and nearest interpolations. (a) PSNR. (b) CNR. (c) NMSE. (d) SSIM. 

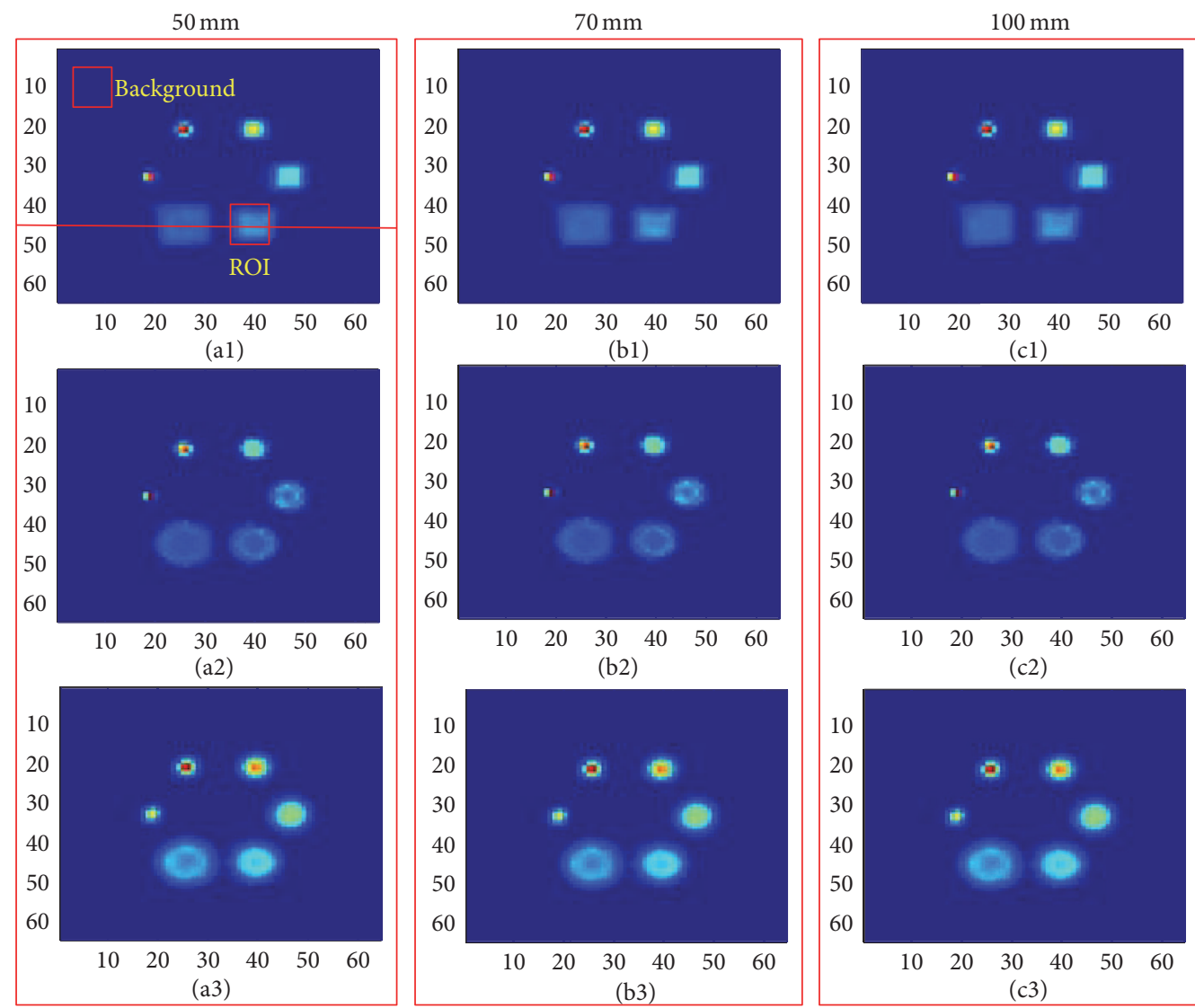

90 degrees

(c2)
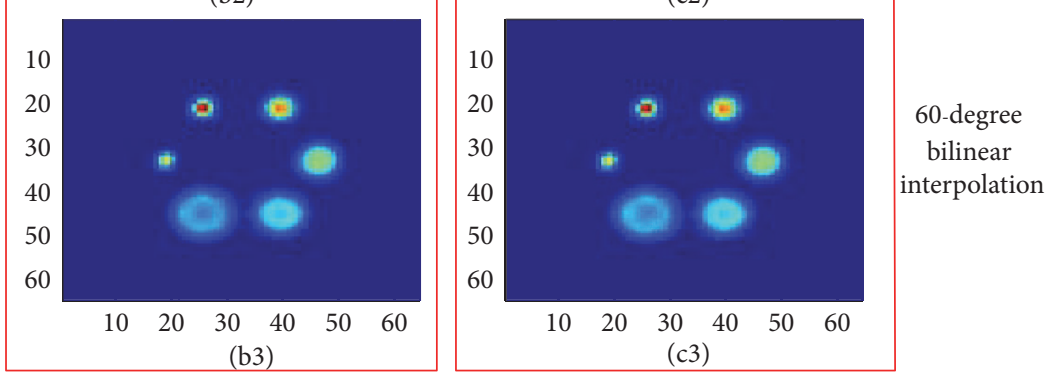

Figure 9: Reconstructed images of the hot spot phantom. The first row is the reconstructed results of $90^{\circ}$ increments. The second and the third row are the results of $60^{\circ}$ increments based on the nearest interpolation and bilinear interpolation, respectively. The columns, from the left to right, show the images from $50 \mathrm{~mm}, 70 \mathrm{~mm}$, and $100 \mathrm{~mm}$, respectively.
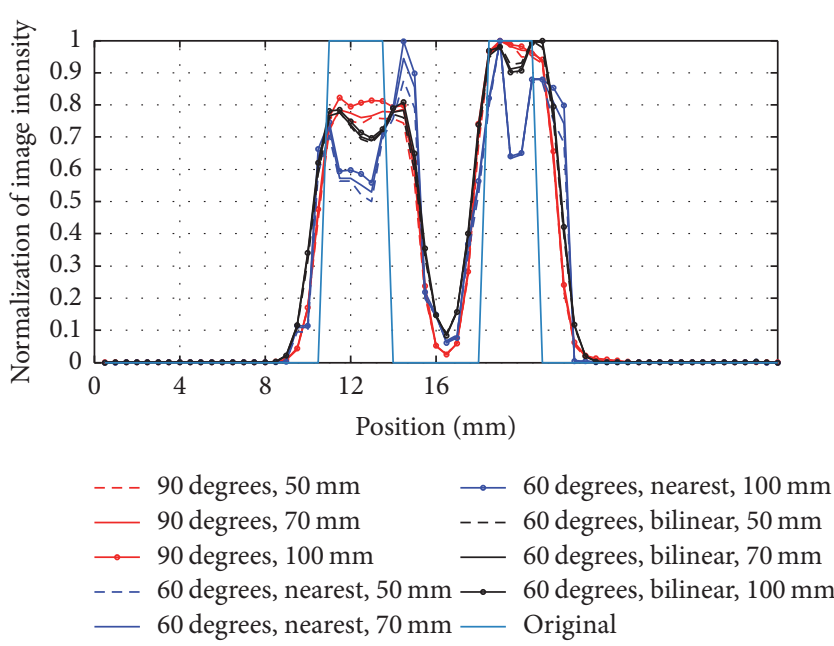

Figure 10: Profiles of normalization of image intensity along the red line in Figure 9.

of $60^{\circ}$ rotation increments had advantages in PSNR, NMSE, and SSIM. In terms of CNR, the reconstructed images of $90^{\circ}$ rotation increments could achieve better results. We also found that the reconstructed images with $90^{\circ}$ rotation increments were uniform by observing. Therefore, both $90^{\circ}$ and $60^{\circ}$ rotation increments could be used in the real experiments, and which one to choose might depend on the application requirement.

3.4. Real Experiment. A phantom with 7 hot points with a diameter of $2 \mathrm{~mm}$ was designed as shown in Figure 11(a). It was made of polymethyl methacrylate (PMMA) and composed of three parts. Each part was $30 \times 30 \times 10 \mathrm{~mm}^{3}$ and contained 3, 2, and 2 hot points, respectively. In the experiment, $20 \mu \mathrm{Ci}{ }^{18} \mathrm{~F}$-FDG solution was poured into these hot points and then the three parts were stacked together. The phantom was placed in the center of the FOV. Considering the size of this phantom, the distance between the detector heads was set to $50 \mathrm{~mm}$. In this situation, the $90^{\circ}$ rotation increments would be enough to maintain the completeness of the forward data; thus, we utilized the $90^{\circ}$ rotation in the experiment. The scanning time lasted $10 \mathrm{~min}$ for each position.

Figures 11(b)-11(e) show the reconstructed images of the phantom. Figures 11(b) and 11(c) are the reconstructed slices of forward data from single angle (nonrotation). Figures 11(d) and $11(\mathrm{e})$ are the results with the $90^{\circ}$ rotatory operation. 


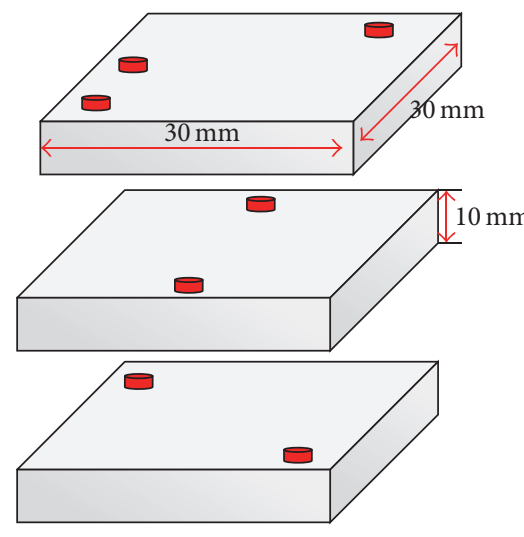

(a)

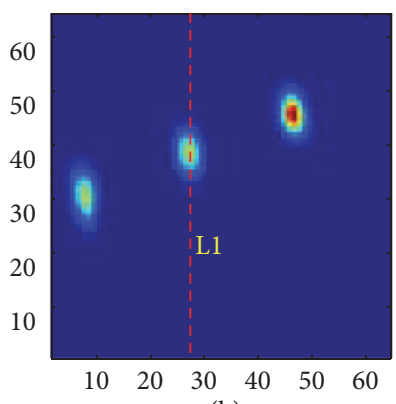

(b)

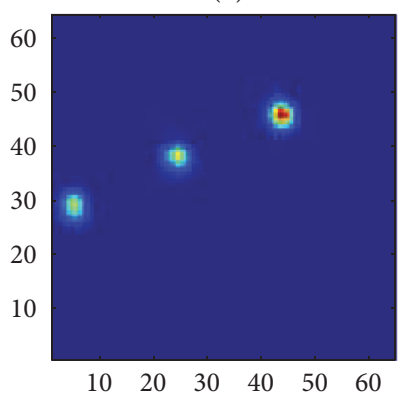

(d)

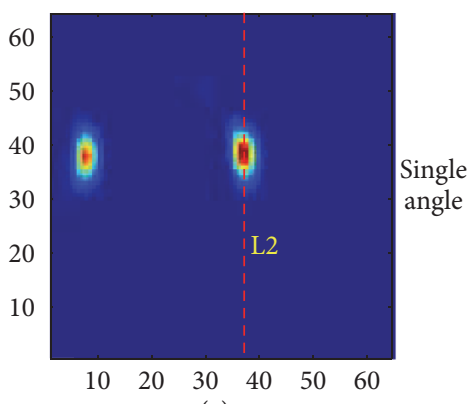

(c)

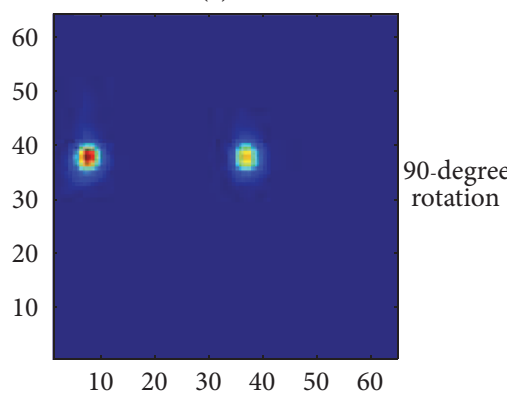

(e)

FIGURE 11: (a) Structure of the phantom. (b) and (c) are the reconstructed slices from single angle (nonrotated). (d) and (e) are the results with the $90^{\circ}$ rotatory operation.

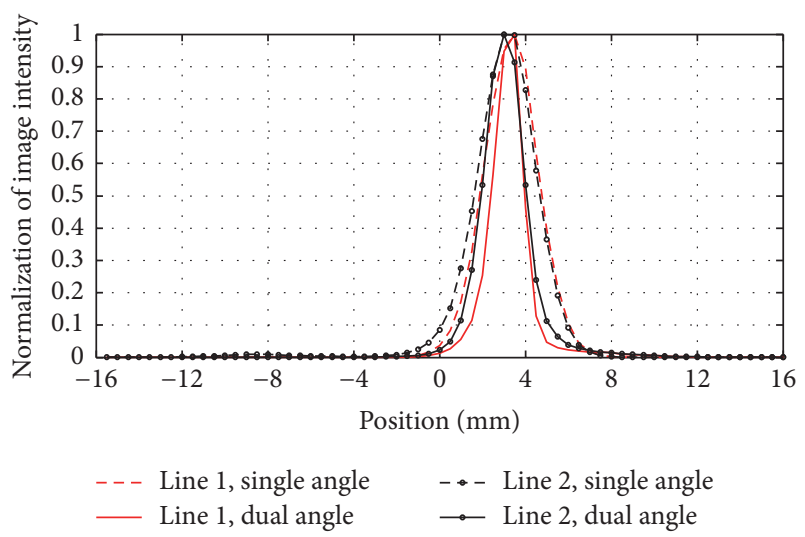

Figure 12: Profiles along the two red lines in Figure 11(b).

The results of a single angle (nonrotation) were taken as a reference. Figure 12 shows the profile along the two red lines in Figures 11(b) and 11(c). By comparing and analyzing the reconstruction results, we could find that the $90^{\circ}$ rotation operation could solve the imaging stretch problem effectively.

\section{Discussion and Conclusion}

In this study, we evaluated the influence of the different rotation increments on imaging performances of a rotatory dualhead PET system. Five rotation increments were compared in the simulation. In the real experiment, we further evaluated the imaging performance of the dual-head PET system with $90^{\circ}$ rotation increments.
In order to fuse the forward data of different acquired positions, a reconstruction flowchart was proposed based on a precalculated SRM which was obtained by MC simulation. For the precalculated SRM, the relationships between the voxels and LORs were fixed. Therefore, we added the image interpolation methods to the reconstruction flowchart. The bicubic interpolation performed worse in both resolution and SSIM as shown in Section 3.1. That may be caused by lots of negative pixels emerging in the images after bicubic interpolation. Those negative pixels are not matched with the OSEM iteration algorithm which has a nonnegativity constraint.

The resolution was evaluated to verify the system's resolving capability without and with rotation operation in Section 3.2. The quantitative and qualitative assessments were evaluated based on the point phantom and the Derenzo phantom, respectively. For the dual-head PET system, the resolutions perpendicular to the detector heads are very low, which can illustrate the effect of the rotation operation. Therefore, only the resolutions in this direction were calculated. By comparing and analyzing the resolution and the reconstructed images, we find that the rotation operation is effective for the dual-head PET system.

The NMSE, PSNR, CNR, and SSIM were applied to evaluate the noise level of the reconstructed images with different rotation increments. In this aspect, the $90^{\circ}$ and $60^{\circ}$ rotation increments had advantages over the other increments. In terms of the NMSE, PSNR, and SSIM, the $60^{\circ}$ rotation increments could obtain the best results. In terms of $\mathrm{CNR}$, the $90^{\circ}$ rotation increments were better than other increments. In addition, the reconstructed images with $90^{\circ}$ rotation increments were uniform as shown in Figures 9 
and 10. Therefore, it is reasonable to take the merits as a reference, and a desired application should also be taken into consideration when determining the rotation increments.

In the simulation experiments, we did not consider the effects of radioactive decays, system dead-time and scatter, or such other physical factors. We also did not consider the effect of mechanical installation. However, those factors cannot be ignored in practice. Therefore, the $90^{\circ}$ rotation operation was conducted in the real experiments. In our future work, we will provide the system performance of a prototype dual-head PET system with $90^{\circ}$ rotation in more detail. A series of in vivo and in vitro experiments will be carried out based on the prototype system.

\section{Competing Interests}

The authors declare that they have no competing interests.

\section{Acknowledgments}

This work was partly supported by the National Natural Science Foundation of China under Grant nos. 81227901, 81571725, 61405149, 61471279, and 81530058, the Natural Science Basic Research Plan in Shaanxi Province of China under Grant no. 2015JZ019, and the Fundamental Research Funds for the Central Universities (NSIZ021402).

\section{References}

[1] A. Del Guerra, A. Bartoli, N. Belcari et al., "Performance evaluation of the fully engineered YAP-(S)PET scanner for small animal imaging," IEEE Transactions on Nuclear Science, vol. 53, no. 3, pp. 1078-1083, 2006.

[2] H. Alva-Sánchez, T. Murrieta, E. Moreno-Barbosa et al., "A small-animal PET system based on LYSO crystal arrays, PSPMTs and a PCI DAQ board," IEEE Transactions on Nuclear Science, vol. 57, no. 1, pp. 85-93, 2010.

[3] H. Zhang, Q. Bao, N. T. Vu et al., "Performance evaluation of PETbox: a low cost bench top preclinical PET scanner," Molecular Imaging and Biology, vol. 13, no. 5, pp. 949-961, 2011.

[4] E. Fysikopoulos, M. Georgiou, N. Efthimiou, S. David, G. Loudos, and G. Matsopoulos, "Fully digital FPGA-based data acquisition system for dual head PET detectors," IEEE Transactions on Nuclear Science, vol. 61, no. 5, pp. 2764-2770, 2014.

[5] W. Luo, E. Anashkin, and C. G. Matthews, "Performance evaluation of a PEM scanner using the NEMA NU 4-2008 small animal PET standards," IEEE Transactions on Nuclear Science, vol. 57, no. 1, pp. 94-103, 2010.

[6] W. W. Moses and J. Qi, "Fundamental limits of positron emission mammography," Nuclear Instruments and Methods in Physics Research Section A: Accelerators, Spectrometers, Detectors and Associated Equipment, vol. 497, no. 1, pp. 82-89, 2003.

[7] C.-M. Kao, J. S. Souris, S. Cho, B. C. Penney, and C.-T. Chen, "Initial performance evaluation of a modular, large-area detector PET scanner for small animal imaging," in Proceedings of the IEEE Nuclear Science Symposium Conference Record, vol. 1-5, pp. 2081-2084, October 2005.

[8] C.-M. Kao, Y. Dong, and Q. Xie, "Evaluation of 3D image reconstruction methods for a dual-head small-animal PET scanner," in Proceedings of the IEEE Nuclear Science Symposium
Conference Record (NSS '07), vol. 4, pp. 3046-3050, IEEE, Honolulu, Hawaii, USA, October 2007.

[9] M. S. Tahaei and A. J. Reader, "Patch-based image reconstruction for PET using prior-image derived dictionaries," Physics in Medicine and Biology, vol. 61, no. 18, pp. 6833-6855, 2016.

[10] L. A. Shepp and Y. Vardi, "Maximum likelihood reconstruction for emission tomography," IEEE Transactions on Medical Imaging, vol. 1, no. 2, pp. 113-122, 1982.

[11] Y. Vardi, L. A. Shepp, and L. Kaufman, "A statistical model for positron emission tomography," Journal of the American Statistical Association, vol. 80, no. 389, pp. 8-20, 1985.

[12] J. Llacer, E. Veklerov, K. J. Coakley, E. J. Hoffman, and J. Nunez, "Statistical analysis of maximum likelihood estimator images of human brain FDG PET studies," IEEE Transactions on Medical Imaging, vol. 12, no. 2, pp. 215-231, 1993.

[13] C. Zhang, X. Chen, S. Zhu, L. Wan, Q. Xie, and J. Liang, "Performance evaluation of a 90॰-rotating dual-head small animal PET system," Physics in Medicine and Biology, vol. 60, no. 15, pp. 5873-5890, 2015.

[14] J. Imrek, D. Novák, G. Hegyesi et al., "Development of an FPGAbased data acquisition module for small animal PET," IEEE Transactions on Nuclear Science, vol. 53, no. 5, pp. 2698-2703, 2006.

[15] J. E. Ortũo, G. Kontaxakis, J. L. Rubio, P. Guerra, and A. Santos, "Efficient methodologies for system matrix modelling in iterative image reconstruction for rotating high-resolution PET," Physics in Medicine and Biology, vol. 55, no. 7, pp. 1833-1861, 2010.

[16] P. Bruyndonckx, C. Lemaître, S. Léonard et al., "Initial characterization of a nonpixelated scintillator detector in a PET prototype demonstrator," IEEE Transactions on Nuclear Science, vol. 53, no. 5, pp. 2543-2548, 2006.

[17] N. Efthimiou, S. Maistros, X. Tripolitis, A. Samartzis, G. Loudos, and G. Panayiotakis, "Tomographic evaluation of a dual-head positron emission tomography system," Measurement Science and Technology, vol. 22, no. 11, Article ID 114010, 2011.

[18] T. Yamaya, E. Yoshida, T. Obi, H. Ito, K. Yoshikawa, and H. Murayama, "First human brain imaging by the jPET-D4 prototype with a pre-computed system matrix," IEEE Transactions on Nuclear Science, vol. 55, no. 5, pp. 2482-2492, 2008.

[19] A. M. Alessio, P. E. Kinahan, and T. K. Lewellen, "Modeling and incorporation of system response functions in 3-D whole body PET," IEEE Transactions on Medical Imaging, vol. 25, no. 7, pp. 828-837, 2006.

[20] P.-E. Danielsson and M. Hammerin, "High-accuracy rotation of images," CVGIP: Graphical Models and Image Processing, vol. 54, no. 4, pp. 340-344, 1992.

[21] S. Jan, G. Santin, D. Strul et al., "GATE: a simulation toolkit for PET and SPECT,' Physics in Medicine and Biology, vol. 49, no. 19, pp. 4543-4561, 2004.

[22] S. Jan, D. Benoit, E. Becheva et al., "GATE V6: a major enhancement of the GATE simulation platform enabling modelling of CT and radiotherapy," Physics in Medicine and Biology, vol. 56, no. 4, pp. 881-901, 2011.

[23] C. M. Kao, Y. Dong, Q. Xie, and C. T. Chen, "Image reconstruction of a dual-head small-animal PET system by using MonteCarlo computed system response matrix," in Proceedings of the 9th International Meeting on Fully Three-Dimensional Image Reconstruction in Radiology and Nuclear Medicine, Lindau, Germany, 2007. 
[24] H. M. Hudson and R. S. Larkin, "Accelerated image reconstruction using ordered subsets of projection data," IEEE Transactions on Medical Imaging, vol. 13, no. 4, pp. 601-609, 1994.

[25] Z. Wang, A. C. Bovik, H. R. Sheikh, and E. P. Simoncelli, "Image quality assessment: from error visibility to structural similarity," IEEE Transactions on Image Processing, vol. 13, no. 4, pp. 600612, 2004.

[26] http://www.ece.uwaterloo.ca/ z70wang/research/ssim/.

[27] NEM Association, "NEMA Standards Publication NU 4-2008, Performance Measurements of Small Animal Positron Emission Tomographs," 2008.

[28] H. Tashima, T. Yamaya, and P. E. Kinahan, "An OpenPET scanner with bridged detectors to compensate for incomplete data," Physics in Medicine and Biology, vol. 59, no. 20, pp. 61756193, 2014.

[29] J. Cheng, D. Tao, Y. Quan et al., "Speckle reduction in 3D optical coherence tomography of retina by A-scan reconstruction," IEEE Transactions on Medical Imaging, vol. 35, no. 10, pp. 22702279, 2016.

[30] J. H. Kim, I. J. Ahn, W. H. Nam, and J. B. Ra, "An effective postfiltering framework for 3-D PET image denoising based on noise and sensitivity characteristics," IEEE Transactions on Nuclear Science, vol. 62, no. 1, pp. 137-147, 2015.

[31] A. L. Goertzen, Y. S. Joon, and C. J. Thompson, "Imaging of weak-source distributions in LSO-based small-animal PET scanners," Journal of Nuclear Medicine, vol. 48, no. 10, pp. 16921698, 2007. 


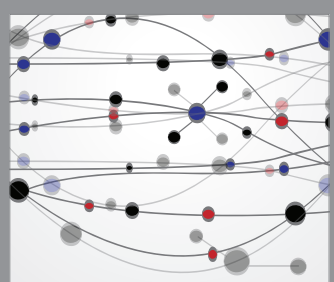

The Scientific World Journal
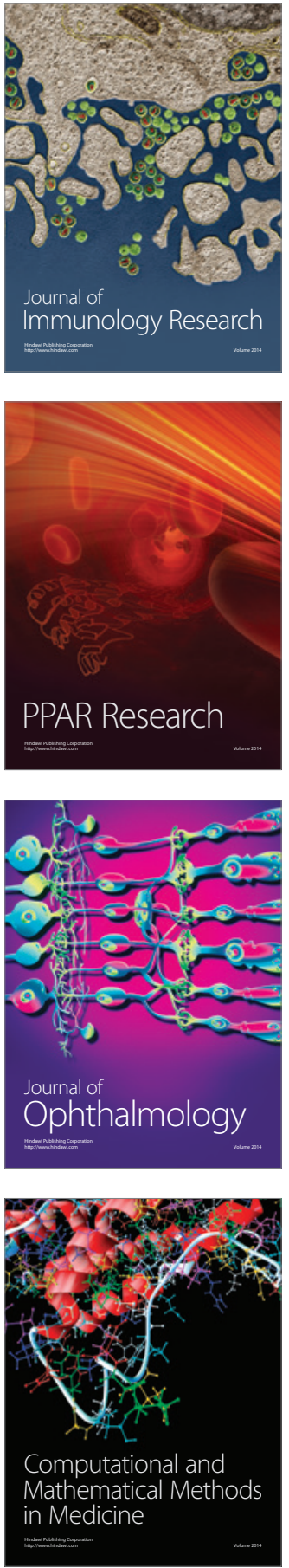

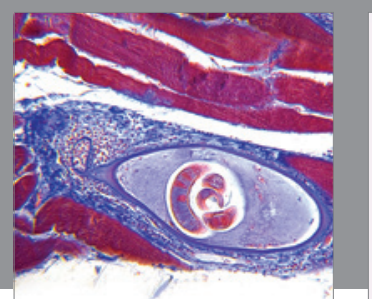

Gastroenterology Research and Practice
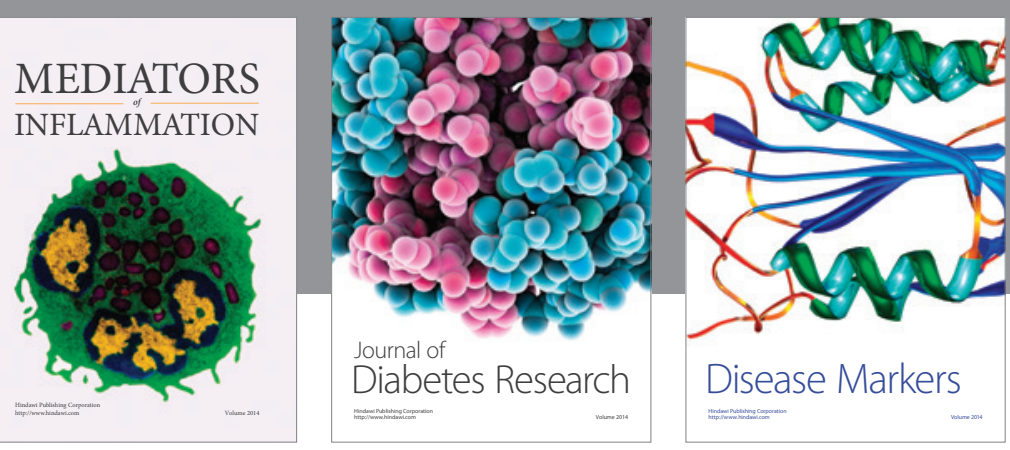

Disease Markers

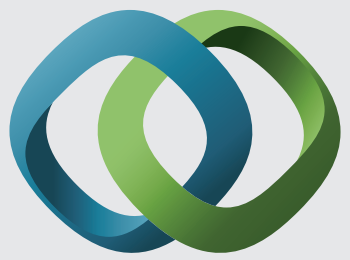

\section{Hindawi}

Submit your manuscripts at

https://www.hindawi.com
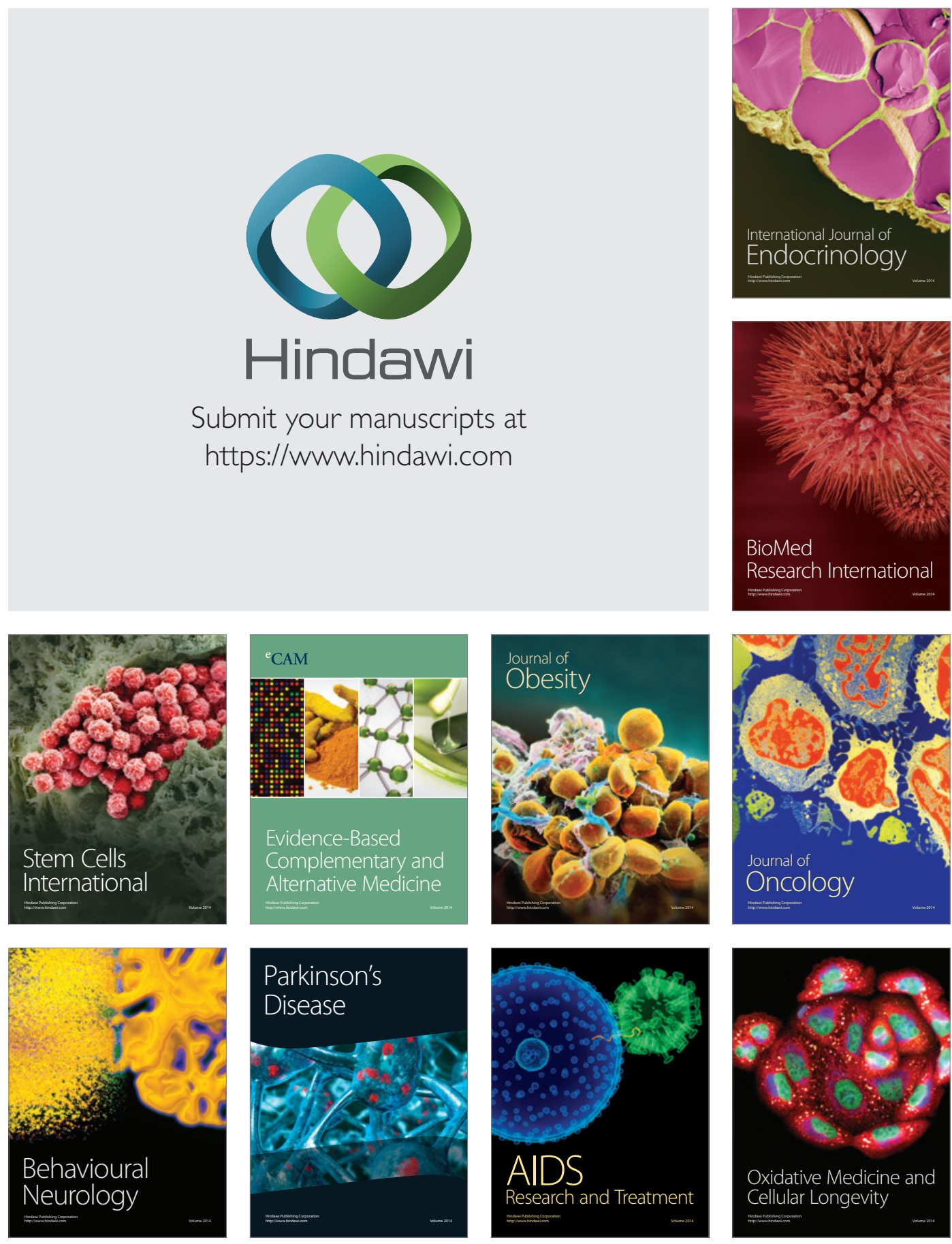